\title{
An exploratory case study of interactive simulation for teaching Ecology
}

\author{
Omair Ameerbakhsh \\ Savi Maharaj \\ Amir Hussain \\ Computing Science and Mathematics, \\ University of Stirling, Stirling, UK \\ oal@cs.stir.ac.uk
}

\author{
Tim Paine \\ Biological and Environmental \\ Sciences, \\ University of Stirling, Stirling, UK \\ c.e.t.paine@stir.ac.uk
}

\author{
Solène Taiksi \\ Centre Condorcet, Université de \\ Bourgogne, France \\ solene_guillaume@etu.u- \\ bourgogne.fr
}

\begin{abstract}
This paper explores the effectiveness of interactive simulation for teaching a selected complex subject, Ecology, in higher education. Specifically, we carry out a lab intervention using interactive agent based simulation, to teach the complex concept of spatially-explicit predator prey interaction to undergraduate students of an advanced module: BIOU9CE (Community Ecology \& Conservation Applications) at the University of Stirling. We propose use of Netlogo, an interactive agent-based simulation tool, and evaluate its effectiveness for learning and teaching of interactive simulation developed specifically for the classroom, compared with an existing, less interactive, simulation tool $(R)$.
\end{abstract}

Index Terms - E-learning, interactive computer simulation, ecology, case study

\section{INTRODUCTION}

Interactive simulation has been increasingly incorporated into science education and has been shown to significantly enhance the teaching-learning process[1]. It has been used in physics, chemistry, biology, mathematics and other sciences [2]. In ecology, it has been reported that computer simulation can improve skills relating to the analysis and application of ecological models [3]. In this research, we investigate the effectiveness of using interactive agent-based computer simulation software (Netlogo[4]) compared to a less interactive software system (R[5]) in teaching ecology to higher education students. A comparison was carried out using 20 undergraduate students taking the BIOU9CE module at the University of Stirling. Results indicate that the students found the NetLogo interactive simulation to be more engaging. In the future, we will extend our exploration of interactive simulation as a learning tool by applying it to other science subjects and with university students from different learning backgrounds.

The aim of this research is to investigate the effectiveness of interactive simulation in teaching
Ecology at higher education level. The investigation was carried out by running interventions in an undergraduate class at the University of Stirling. We compare the preexisting teaching method (non-interactive simulation in R) with an interactive simulation we developed specifically for these classes.

This paper is organized as follows: Section II describes the spatially-explicit predator prey model which is used to investigate the effectiveness of interactive simulation in teaching Ecology to an undergraduate class at the University of Stirling. Section III presents the proposed interactive agent-based simulation tool (Netlogo) and the research methodology used to run interventions. Section IV discusses the findings from comparing the use of Netlogo with a pre-existing teaching method (noninteractive simulation in R). Finally, some concluding remarks and future work suggestions are given in Section $\mathrm{V}$.

\section{SPATIALLY-EXPLICIT PREDATOR PREY LAB PRACTICAL MODEL}

The purpose of the classroom exercise is for the student to learn about predator-prey interaction by using a realistic predator-prey model. In so doing, the student will:

1. explore the linkages between ecological processes and their representations in models

2. explore how explicitly accounting for space affects the outcome of models

3. explore ways to use models to predict the outcome of predator-prey interactions

4. design and execute a modeling study of predatorprey dynamics.

The model considers how space affects the interaction between individuals and their ecology. Populations have spatial structure because individuals are located at specific locations in space. This has several effects on their ecology. First, an individual's spatial location restricts the set of organisms that it can interact with to be 
those in its local neighborhood. Second, space (together with the sensory organs of the organism in question) affects the detectability of predators and prey. Third, heterogeneity in the spatial distribution of resource availability, refuges, mates and abiotic conditions, etc., can strongly influence ecological processes. Finally, the viscosity of the environment, together with the dispersal abilities of the organism, affects how quickly they can move through space. All three of these factors influence ecological interactions among organisms.

\section{Modeling platforms}

In the lab practical we used two modeling platforms, $\mathrm{R}$ and NetLogo, in which the model described above has been developed. The primary motivation is to provide a user-friendly way to interact with a relatively detailed model. This feeds into the secondary motivation, which is to evaluate the educational potential of $\mathrm{R}$ and NetLogo. This will help improve teaching provision in future years. NetLogo is a multi-agent programmable modeling environment used by tens of thousands of students, teachers and researchers worldwide. Models are written in the NetLogo language and NetLogo provides a graphical user interface for model users.

\section{Description of model}

ARENA: Predator-prey dynamics are simulated within a homogeneous, two-dimensional closed habitat. The habitat is rectangular, with dimensions specified by the student. The model is spatially explicit, with each individual having a set location in the habitat. In $\mathrm{R}$, space is continuous and individuals occupy $\mathrm{X}-\mathrm{Y}$ coordinates. In NetLogo, individuals occupy grid cells, but the grid is so fine that space is effectively continuous. In both $\mathrm{R}$ and NetLogo, by joining the top and bottom edges of the arena, or the left and right edges, we can create either a vertical (or horizontal) cylinder. By joining both the top/bottom and the left/right edges, we create a torus (a donut). These manipulations make the spatial area of simulation essentially endless.

TIME: Time is discrete, with a small step size.

MOVEMENT: Prey move throughout the habitat at a speed determined by the student (Nspeed). They move in randomly-chosen directions, unless there is a predator within a 'dodge_radius', in which case they move away from the nearest predator (with a certain degree of error). Predators, likewise, move at a speed determined by the student (Pspeed). Again, they move randomly unless they are within a 'search_radius' of prey, in which case they move towards the nearest prey (again, with a certain degree of error). When a prey is located within a certain 'catch_dist' of a predator it is considered to be caught and eaten by that predator. If several predators catch a prey simultaneously, they share it. We assume that all prey contain the same level of resources, as far as the predator is concerned.

GROWTH: Prey grow by acquiring resources from the environment. There is density dependent competition among prey, however. We assess how many other prey are present in the neighborhood around each individual. Elevated local density reduces the resource gain for each affected prey. This effect is modulated by the parameter 'dd' (density dependence). When dd is high, the effect of local crowding is particularly severe. Predators, on the other hand, grow by consuming prey.

REPRODUCTION: Prey and predators must obtain a threshold level of resources from the environment in order to reproduce. Reproduction is by asexual budding: each new individual is generated at the same location as the parent, with a minimal level of resources. The threshold levels of resources necessary for reproduction by prey and predators (' $\mathrm{Nb}$ ' and ' $\mathrm{Pb}$ ') are set by the user.

DEATH: Mortality for the prey occurs only when they are consumed by the predator. Predators face a user-defined per-capita probability of death (d) in every time-step.

INITIAL CONDITIONS: The used indicates how many predators and prey are initially present in the arena. They are located randomly, with a random energy level.

\section{Model outputs}

The main model output is stability, measured as the persistence of the two species to the 'max.time' (a variable that you can adjust). We also obtain from the model the mean population size of the prey and predators, as well as their ranges, which gives an indication of the amplitude of variation in population sizes. Greater oscillations, and oscillations that intensify through time, are indicators of instability, whereas small and damped oscillations indicate relative stability.

In addition to population dynamics, students can observe the spatial patterning of predators and prey in the arena are they all spread out? Do predators hunt as a group? Do prey disperse from one another and from predators? 


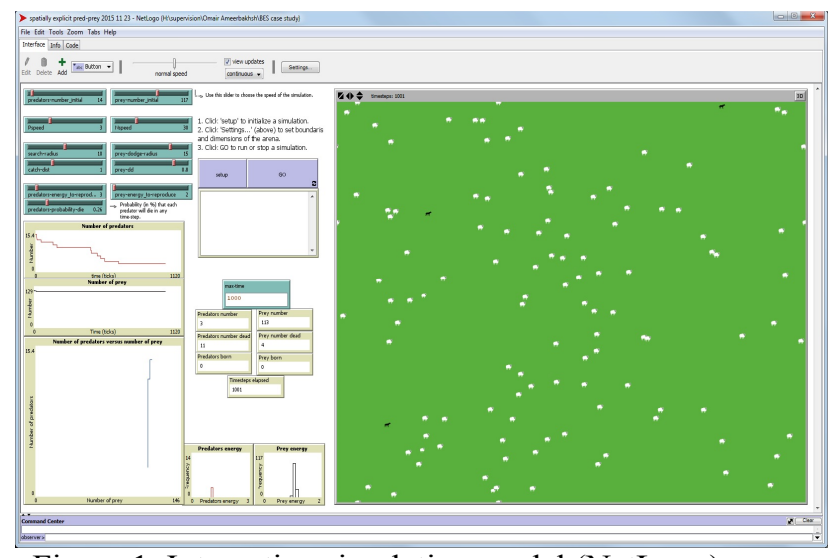

Figure 1. Interactive simulation model (NetLogo).

In NetLogo (Figure 1), prey are shown as white sheep and predators as black wolves, whereas in R (Figure 2), prey are black circles and predators are red stars. In both, their size indicates their current level of resources. In R, the heavy black bar on the right indicates the 'catch distance' of the predator. Any prey within this distance of a predator dies. The thin red line indicates the dodge radius of the prey. If there are predators within this distance, prey will try to avoid them. The tall thin bar at the bottom right indicates the progress of the simulation up to the maximum number of time-steps specified by the user. This simulation run just ended, with 102 prey and 91 predators alive at the end. Similar information is provided in NetLogo's graphical user interface.

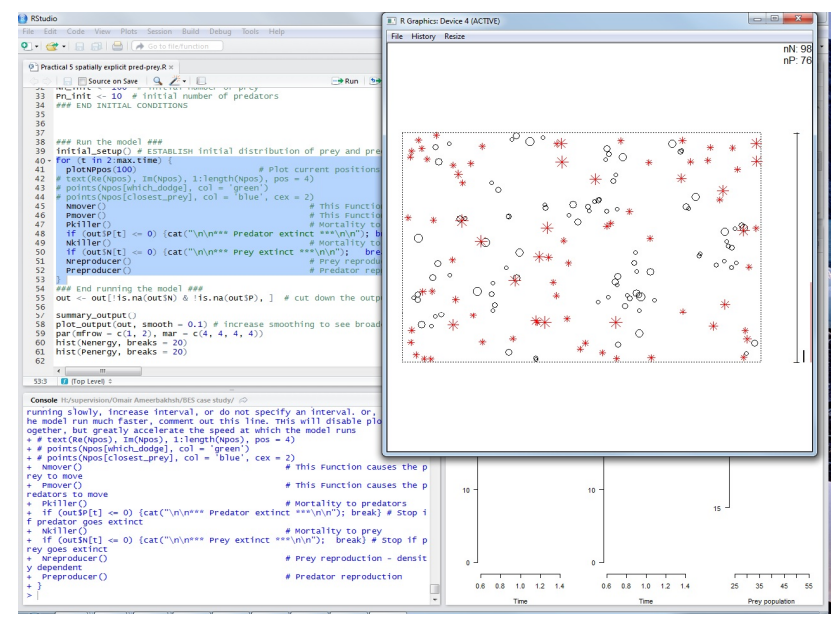

Figure 2. Non Interactive simulation model (R).

\section{Manipulations}

Students used the system to investigate a wide variety of ecological hypotheses. For example, they might hypothesize that:

- the probability of prey survival decreases as their speed decreases and the predator speed increases;
- increasing the predator's search radius decreases the probability of stable coexistence, whereas decreasing the search radius increases it. Changes in the dodge radius would have the contrary effect;

- changing the resources needed for reproduction for predator and prey would affect their population sizes and stability;

- changing the surface area-perimeter ratio would affect the stability of coexistence.

Many additional manipulations are possible. We settled on the above manipulations due to lab time constraints.

\section{Interactive Simulation (NetLogo)}

The main focus of this research is on using interactive agent-based simulation software (Netlogo) to teach complex subjects such as ecology to university students. Interactive simulation is a representation of an event or procedure, in which the outcome is changeable by the user[6]. NetLogo is a modelling environment and multiagent programming language for simulating complex natural and social phenomena. It is good at modelling complex evolving systems. Models can instruct hundreds or thousands of "agents" to explore the micro-level behaviour of individuals and macro-level patterns that emerge. NetLogo allows users to modify simulations to explore their behaviour in different scenarios. NetLogo is simple enough for students and researchers who are not programmers to create their own models. NetLogo is a standalone Java application which can run on all major computing platforms [7].

The term simulation is described as an artificial environment that is carefully built to manage individuals' experience of reality[8]. It works as an exercise implicating reality of function but in a simulated environment[9].

Interactive computer simulations provide many benefits to support calls for inquiry-based, learner and knowledgecentered teaching and instruction[10]. For example, simulations offer the benefit of flexibility, supporting students to actively engage in problem-solving, higherorder thinking and in reinforced practice[11]. Therefore, interactive computer simulations have the potential to make teaching more interactive and make learning abstract concepts more real. Interactive computer simulation let students challenge their own theories by working with and receiving immediate feedback about original and/or real data and making tailored problemsolving decisions [12].

\section{Methodology}

Our basic research methodology is inductive enquiry, as there are no existing frameworks for studying the effectiveness of interactive simulation for teaching 
ecology in higher education. The approach used is a mixed method (quantitative + qualitative) strategy [13]. The quantitative part uses learning effectiveness surveys (LES)[14][15] to evaluate students' responses to the two simulation tools, and a self-efficacy scale [16] to measure the students' perception of their capability to run and manipulate each simulation. These were applied in a randomized controlled crossover lab intervention setup [17]. We chose this structure for ethical reasons, to give all students the chance to use both interactive and noninteractive simulation models. The intervention took place in two stages, with students divided into two groups A and B. In the first stage, group A carried out an exercise using a non-interactive $\mathrm{R}$ simulation and group $\mathrm{B}$ did the same exercise using an interactive NetLogo simulation. In the second stage, group A did the exercise using NetLogo and group B used R. Learning effectiveness questionnaires were applied after each stage. At the end of the intervention, students were given opinion questionnaires for feedback on the two simulation tools to collect qualitative data. The questionnaires include questions about preferences, reasons for preferences, effectiveness, power, advantages and disadvantages of both tools. Finally, a semi-structured interview was conducted with the lecturer.

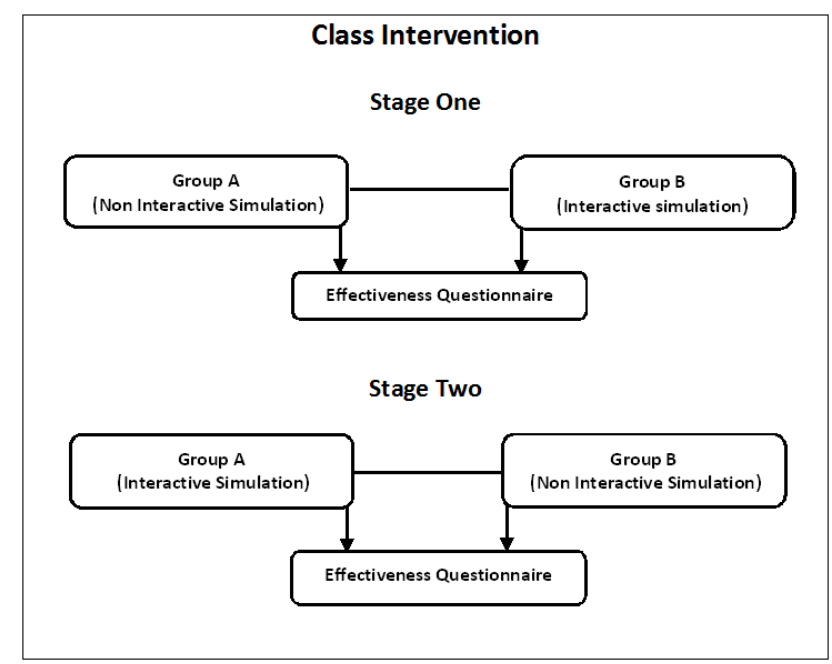

Figure 3: Class Intervention.

\section{Learning Effectiveness Survey Questions}

To what extent do you feel that you have learned from this version of the model in today's lab practical?

To what extent do you feel that the model could help you more to explore the linkages between ecological processes and their representations in models?

To what extent do you feel that the model could help you to better explore how explicitly accounting for space affects ecological interactions?
To what extent do you feel that the model could help you more to explore ways to predict the outcome of predator-prey interactions?

To what extent do you feel that the model could help you more to design and execute a modelling study of predator-prey dynamics?

How effective was this version of the model at helping you learn the key concepts?

How easy was this version of the model to use?

How engaging did you find the exercise using this version of the model?

How visually attractive did you find the user interface in this version of the model?

How much did this version of the model help you understand the spatially-explicit predator prey concept?

How able were you to manipulate this version of the model, as requested in the lab handout?

How capable were you to evaluate the first suggested hypothesis: "the probability of prey survival decreases as their speed decreases and the predator speed increases"?

How capable were you to evaluate the second suggested hypothesis: "increasing the predator's search radius decreases the probability of stable coexistence, whereas decreasing the search radius increases it..."?

How capable were you to investigate the third suggested hypothesis: "changing the resources needed for reproduction for predator and prey would affect their population sizes and stability"?

How enthusiastic were you about using this version of the model?

How much do you feel that this version of the model will help you in completing your assignment?

Table 1: Learning Effectiveness Survey Questions

At the end of the lab students were given an assignment to choose one or more ecological hypotheses to test using the modeling platform of their choice. There are many hypotheses that they can examine. Each student was advised to test hypotheses that are different from colleagues. They were also advised to consider what parameters of the model they will manipulate, and what response variables they will measure to evaluate their hypotheses. Also, to consider how they will replicate their study to obtain confidence in their results. They were asked to consult with the instructor about their study design prior to testing their hypotheses. 17 students out the 20 chose NetLogo for their assignment and the remaining 3 students chose $R$. This suggests that students preferred the interactive simulation model over the noninteractive simulation model.

\section{RESUlTS AND ANALYSIS}

The learning effectiveness survey used a Likert scale of 6 score where $1=$ nothing or not at all and $6=\mathrm{a}$ lot or 
very much. There were fewer than 50 participants; it is therefore appropriate to use nonparametric tests such as the Mann-Whitney-Wilcoxon U Test to analyse the data [18] [19].

Ranks

\begin{tabular}{|l|l|l|l|l|}
\hline & Software & $\mathrm{N}$ & $\begin{array}{l}\text { Mean } \\
\text { Rank }\end{array}$ & Sum of Ranks \\
\hline Total & Netlogo & 9 & 13.72 & 123.50 \\
R & 9 & 5.28 & 47.50 \\
Total & 18 & & \\
\hline
\end{tabular}

Test Statistics ${ }^{\mathrm{a}}$

\begin{tabular}{|l|l|}
\hline & Total \\
\hline Mann-Whitney U & 2.500 \\
Wilcoxon W & 47.500 \\
$\mathrm{Z}$ & -3.368 \\
Asymp. Sig. (2-tailed) & .001 \\
Exact Sig. [2*(1-tailed Sig.)] & $.000^{\mathrm{b}}$ \\
\hline
\end{tabular}

Table 2: Results of Mann-Whitney U test for the first stage of the intervention.

In the first stage of the intervention, the Mann-Whitney $\mathrm{U}$ test indicates that the survey total score was greater for Group B, who used NetLogo (median $=84$ ), than for Group A, who used R (median $=55), \mathrm{U}=2.500, p=.000$. This means that in this stage, the scores of students who used NetLogo were higher than those of students who used $\mathrm{R}$, and therefore interactive simulation model seems the preferred choice in this stage.

\begin{tabular}{|l|l|l|l|l|l|}
\hline & Q6 & Q7 & Q8 & Q9 & Q10 \\
\hline $\begin{array}{l}\text { Mann-Whitney } \\
\text { U }\end{array}$ & 3.000 & 4.500 & 16.500 & 8.500 & 13.000 \\
Wilcoxon W & 48.000 & 49.500 & 61.500 & 53.500 & 58.000 \\
Z & -3.393 & -3.293 & -2.163 & -2.937 & -2.532 \\
Asymp. Sig. (2- & .001 & .001 & .031 & .003 & .011 \\
tailed) & & & & & \\
$\begin{array}{l}\text { Exact Sig. } \\
\text { [2*(1-tailed }\end{array}$ & $.000^{\mathrm{b}}$ & $.000^{\mathrm{b}}$ & $.031^{\mathrm{b}}$ & $.003^{\mathrm{b}}$ & $.014^{\mathrm{b}}$ \\
Sig.)] & & & & & \\
\hline
\end{tabular}

Table 3: Test Statistics for student's scores for questions 6-10 in the first stage.

Table 3 shows that the students' scores for Q6: To what extent do you feel that you have learned from this version of the model in today's lab practical? $(1=$ nothing, $6=$ a lot) from group $B$ who used Netlogo was statistically significantly higher than the $\mathrm{R}$ response from group A $(U=3.000, p=.000)$. For Q7: To what extent do you feel that the model helped you to explore the linkages between ecological processes and their representations in models? $(1=$ not at all, $6=$ very much) group B Netlogo's response was statistically significantly higher than the $\mathrm{R}$ response from group $\mathrm{A}$ $(U=4.500, p=.000)$. For Q8 To what extent do you feel that the model helped you to explore how explicitly accounting for space affects ecological interactions? $(1=$ not at all, $6=$ very much $)$ group $B$ Netlogo's response was statistically significantly higher than the $\mathrm{R}$ response from group $\mathrm{A}(U=16.500, p=.031)$. For Q9 To what extent do you feel that the model helped you to explore ways to predict the outcome of predator-prey interactions? $(1=$ not at all, $6=$ very much) group B Netlogo's response was statistically significantly higher than the $\mathrm{R}$ response from group $\mathrm{A}$ $(U=8.500, p=.003)$. For Q10 To what extent do you feel that the model helped you to design and execute a modelling study of predator-prey dynamics? $(1=$ not at all, 6 = very much) group B Netlogo's response was statistically significantly higher than the $\mathrm{R}$ response from group $\mathrm{A}(U=13.000, p=.014)$.

\begin{tabular}{|c|c|c|c|c|}
\hline \multicolumn{5}{|c|}{ Ranks } \\
\hline & Software & $\mathrm{N}$ & $\begin{array}{l}\text { Mean } \\
\text { Rank }\end{array}$ & $\begin{array}{l}\text { Sum of } \\
\text { Ranks }\end{array}$ \\
\hline \multirow[t]{3}{*}{ total } & Netlogo & 9 & 13.06 & 117.50 \\
\hline & $\mathrm{R}$ & 9 & 5.94 & 53.50 \\
\hline & Total & 18 & & \\
\hline
\end{tabular}

Test Statistics ${ }^{\mathbf{a}}$
\begin{tabular}{|l|l|}
\hline & total \\
\hline Mann-Whitney U & 8.500 \\
Wilcoxon W & 53.500 \\
$\mathrm{Z}$ & -2.830 \\
Asymp. Sig. (2-tailed) & .005 \\
Exact Sig. [2*(1-tailed Sig.)] & $.003^{\mathrm{b}}$ \\
\hline
\end{tabular}

Table 4: Results of Mann-Whitney U test for the second stage of the intervention

In the second stage, the Mann-Whitney $U$ test indicates that the survey total score for Group A, who used NetLogo in this stage, was greater $($ median $=78)$ than for Group B, who used R (median $=60), \mathrm{U}=8.500, p=.003$. This means that in this stage, the scores of students who used NetLogo were higher than those of students who 
used $\mathrm{R}$, and therefore interactive simulation again seems the preferred choice.

We also used the Mann-Whitney U test to analyse the results of the self-efficacy scale, which measured the students' perception of their capability to run and manipulate the simulation. There was no significant difference between the scores for R and Netlogo.

For the opinion questionnaire and lecturer interview we used framework based thematic analysis [9][10] to code the data. In the feedback survey, out of 18 respondents, 17 preferred the interactive simulation and thought it to be more effective. 13 students believed that $\mathrm{R}$ was more powerful than Netlogo. We speculate that this may be because the students had not used Netlogo before and lack knowledge of its advanced features. 13 students said that they would choose Netlogo over R if given the choice. 10 students said that Netlogo is easier to use for reasons such as: interaction, clarity, user friendliness, better presentation and visualization. The lecturer found the Netlogo model useful, approachable, attractive and effective for teaching complex ecological models. He thought that $\mathrm{R}$ was more powerful but stated that his response was based on regular use of $\mathrm{R}$ and unfamiliarity with Netlogo. He also thought that students responded very positively to Netlogo and found it very effective.

\begin{tabular}{|l|l|}
\hline Theme & Implication \\
\hline Game & $\begin{array}{l}\text { Netlogo is a toy program. Looks like a } \\
\text { game. }\end{array}$ \\
\hline Approachable & $\begin{array}{l}\text { Not intimidating to for students who did } \\
\text { not have a lot of experience in using } \\
\text { Netlogo before. }\end{array}$ \\
\hline Interactive & $\begin{array}{l}\text { Netlogo parameter can be adjusted } \\
\text { during the running of the simulation. }\end{array}$ \\
\hline Dynamic & $\begin{array}{l}\text { Ability to make multiple graphs during } \\
\text { run of the simulation. }\end{array}$ \\
\hline Abstraction & $\begin{array}{l}\text { Students will never have to the code and } \\
\text { they can deal with the graphical user } \\
\text { interface not like in the R model. }\end{array}$ \\
\hline Effective & $\begin{array}{l}\text { Students were very engaged with it and } \\
\text { managed to complete all manipulations } \\
\text { and enjoyed using as a game based } \\
\text { learning tool. }\end{array}$ \\
\hline
\end{tabular}

Table 5: Thematic analysis of teacher interview

\section{CONCLUSION}

The study showed that our proposed interactive agentbased simulation with NetLogo was preferred by both students and the lecturer as the teaching and learning tool for the complex Ecology class exercise. It allowed learners to interact and engage with a simulation more than the non-interactive simulation, and helped the students to learn the complex ecological model in an easy and enjoyable way, with some students describing it as a game. We plan to use interactive simulation in other fields such as Aquaculture and with bigger groups to explore the effectiveness of using interactive simulation in teaching a range of complex subjects to university students.

\section{REFERENCES}

[1] M. Ceberio, J. M. Almudí, and Á. Franco, "Design and Application of Interactive Simulations in ProblemSolving in University-Level Physics Education," J. Sci. Educ. Technol., 2016.

[2] W. K. Adams, "Student engagement and learning with PhET interactive simulations," Nuovo Cim. della Soc. Ital. di Fis. C, vol. 33, no. 3, pp. 21-32, 2010.

[3] K. Korfiatis, E. Papatheodorou, G. P. Stamou, and S. Paraskevopoulous, "An investigation of the effectiveness of computer simulation programs as tutorial tools for teaching population ecology at university," Int. J. Sci. Educ., vol. 21, no. 12, pp. 1269 1280, 1999.

[4] U. Wilensky, "NetLogo (and NetLogo User Manual), Center for Connected Learning and Computer- Based Modeling," 1999.

[5] W. Venables and D. Smith, "An Introduction to R Notes on R: A Programming Environment for Data Analysis and Graphics Version 3.3.0", 2016.

[6] M. Stieff and U. Wilensky, "Connected Chemistry, Incorporating Interactive Simulations into the Chemistry Classroom," J. Sci. Educ. Technol., vol. 12, no. 3, p. 285, 2003.

[7] S. Tisue and U. Wilensky, "NetLogo: A Simple Environment for Modeling Complexity," in The International Conference on Complex Systems, 2004, pp. 1-10.

[8] B. S. Bell, A. M. Kanar, and S. W. J. Kozlowski, "Current issues and future directions in treatment," Int. J. Hum. Resour. Manag., vol. 19, no. 8, pp. 416-1434, 2008.

[9] K. Jones, "Who's Who, Who Sits Where," Hisp. J. Behav. Sci., vol. 29, no. 3, pp. 328-330, 1998.

[10] NRC, National Science Education Standards. WASHINGTON, DC: NATIONAL ACADEMY PRESS, 1996.

[11] J. Hargrave, C., \& Kenton, "Preinstructional simulations: Implications for science classroom teaching," J. Comput. Math. Sci. Teach., vol. 19(1), pp. 47-58, 2000.

[12] L. K. Smetana and R. L. Bell, "Computer Simulations to Support Science Instruction and Learning : A critical review of the literature Computer Simulations to Support Science Instruction and Learning: A critical review of the literature," Int. J. Sci. Educ., vol. 34, no. 9, pp. 1337-1370, 2012.

[13] H. Aramo-immonen, "Mixed Methods Research Design," Springer-Verlag Berlin Heidelb., pp. 32-43, 2013.

[14] G. Moody, D. L., \& Sindre, "The Learning Effectiveness Survey (LES): An instrument for evaluating and improving the effectiveness of learning interventions," in Hawaii International Conference on Education, 2003, pp. pp. 7-10.

[15] D. L. Moody and G. Sinder, "Evaluating the 
Effectiveness of Learning Interventions: An Information Systems Case Study," in ECIS 2003 Proceedings, 2003, p. 17.

[16] A. Bandura, "Guide for constructing self-efficacy scales," in Self-efficacy beliefs of adolescents, Information Age Publishing, 2006, pp. 307-337.

[17] M. K. Turner, S. R. Simon, K. C. Facemyer, L. M. Newhall, and T. L. Veach, "Teaching and Learning in Medicine: An International Web-Based Learning Versus Standardized Patients For Teaching Clinical Diagnosis : A Randomized, Controlled, Crossover Trial Web-Based Learning Versus Standardized Patients For Teaching Clinical Diagnosis," Teach. Learn. Med. An Int. JournalJournal, vol. 18, no. 3, pp. 208-241, 2010.

[18] J. C. F. de Winter and D. Dodou, "Five-Point Likert Items : t test versus Mann-Whitney-Wilcoxon," Pract. Assessment, Res. Eval., vol. 15, no. 11, pp. 1-16, 2010.

[19] N. Nachar, "The Mann-Whitney U: A Test for Assessing Whether Two Independent Samples Come from the Same Distribution," Tutor. Quant. Methods Psychol., vol. 4, no. 1, pp. 13-20, 2008. 